\title{
Nonlinear analysis of electromyogram following gait training with myoelectrically triggered neuromuscular electrical stimulation in stroke survivors
}

Anirban Dutta ${ }^{1 *}$, Bhawna Khattar ${ }^{2}$ and Alakananda Banerjee ${ }^{2}$

\begin{abstract}
Neuromuscular electrical stimulation (NMES) facilitates ambulatory function after paralysis by activating the muscles of the lower extremities. The NMES-assisted stepping can either be triggered by a heel-switch (switch-trigger), or by an electromyogram (EMG)-based gait event detector (EMG-trigger). The command sources—switch-trigger or EMG-trigger-were presented to each group of six chronic ( $>6$ months post-stroke) hemiplegic stroke survivors. The switch-trigger group underwent transcutaneous NMES-assisted gait training for $1 \mathrm{~h}$, five times a week for 2 weeks, where the stimulation of the tibialis anterior muscle of the paretic limb was triggered with a heel-switch detecting heel-rise of the same limb. The EMG-trigger group underwent transcutaneous NMES-assisted gait training of the same duration and frequency where the stimulation was triggered with surface EMG from medial gastrocnemius (MG) of the paretic limb in conjunction with a heel-switch detecting heel-rise of the same limb. During the baseline and post-intervention surface EMG assessment, a total of $10 \mathrm{~s}$ of surface EMG was recorded from bilateral MG muscle while the subjects tried to stand steady on their toes. A nonlinear tool-recurrence quantification analysis (RQA) — was used to analyze the surface EMG. The objective of this study was to find the effect of NMES-assisted gait training with switch-trigger or EMG-trigger on two RQA parameters - the percentage of recurrence (\%Rec) and determinism (\%Det), which were extracted from surface EMG during fatiguing contractions of the paretic muscle. The experimental results showed that during fatiguing contractions, (1) \%Rec and \%Det have a higher initial value for paretic muscle than the non-paretic muscle, (2) the rate of change in \%Rec and \%Det was negative for the paretic muscle but positive for the non-paretic muscle, (3) the rate of change in \%Rec and \%Det significantly increased from baseline for the paretic muscle after EMG-triggered NMES-assisted gait training. Therefore, the study showed an improvement in paretic muscle function during a fatiguing task following gait training with EMG-triggered NMES. This study also showed that RQA parameters—\%Rec and \%Det—were sensitive to changes in paretic/non-paretic muscle properties due to gait training and can be used for non-invasive muscle monitoring in stroke survivors undergoing rehabilitation.
\end{abstract}

Keywords: Neuromuscular electrical stimulation, Electromyogram, Foot-drop, Stroke, Recurrent quantification analysis

\footnotetext{
* Correspondence: anirban.dutta@med.uni-goettingen.de

'Department of Clinical Neurophysiology, Georg-August-University, Goettingen, Germany

Full list of author information is available at the end of the article
} 


\section{Introduction}

The World Health Organization defines stroke as "the rapid development of clinical signs and symptoms of a focal neurological disturbance lasting more than $24 \mathrm{~h}$ or leading to death with no apparent cause other than vascular origin" [1]. Worldwide, 15 million people suffer a stroke every year, and stroke is the second leading cause of disability after dementia. India-centered studies have presented a prevalence rate of $27-34 / 100,000$ in the $35-44$ age group to $822-1116 / 100,000$ in the $75+$ age group [2-4]. The prevalence of stroke in younger individuals is higher in India as compared with high-income nations [5]. The burden of stroke on the quality of life is much greater in this younger age group of stroke survivors [5-7]. Moreover, stroke drastically affects the economical productivity for that younger age-group adding further to the overall disease burden [5] and therefore early rehabilitation to alleviate disability has a special significance in developing countries.

The disability due to poor ability to walk in stroke patients is frequently caused by the "foot-drop" symptom that prevents the patient from being able to raise the foot during the swing phase of walking [8]. The foot slaps down on the ground after heel-strike and the toe drags during the swing phase. In addition to that the patients often lack from push-off power due to weakness in ankle plantarflexors (like, gastrocnemius muscle), which not only reduces walking speed, but also reduce ankle dorsiflexion angles during the early swing phase of gait [9]. Goldberg et al. [10] have shown that the ankle plantarflexors are important for knee flexion velocity during push-off. This leads to insufficient foot clearance that puts the patient at risk for stumbling and falling [9]. Consequently, impairment of walking has been mentioned most frequently (39-90\%) as the most important disabling condition in community-dwelling stroke survivors and improving walking ability has been found to be the best way to reduce dependency $[11,12]$. Almost 30\% of patients who survive stroke are affected by foot-drop, where the ankle dorsiflexors (e.g., tibialis anterior muscle) are functionally impaired $[8,9]$.

Neuromuscular electrical stimulation (NMES)-based orthosis has been shown to enhance walking abilities, increasing gait speed while lowering effort and has only recently developed into a therapeutic intervention for stroke rehabilitation [8,13-22]. NMES involves electrical stimulation of nerves and muscles with continuous short pulses of electrical current [23]. Hausdorff and Ring [20] showed that the number of falls reduced significantly in hemiparetic patients who used FES to correct foot-drop. Although FES provides orthotic benefit but the neurophysiological therapeutic benefit has not been investigated thoroughly. Recently, different command modalities are available where NMES-assisted stepping can either be triggered with a heel-switch (switch-trigger) or with an electromyogram (EMG)-based gait event detector (EMG-trigger). Although both the modalities provide similar orthotic benefit $[24,25]$ where they assist in lifting the foot to clear the ground during stepping action but the relative longterm therapeutic benefit of one modality over the other in facilitating recovery of neurophysiological function has not rigorously been shown. Preliminary research demonstrated the feasibility of EMG control of NMES in partial paralysis as well as its therapeutic benefits [26-31]. Dutta et al. [31] have shown therapeutic benefits in terms of incremental improvement in the classification of the gait event using a constant EMG-based classifier while the command muscle was undergoing NMES-assisted gait training. However, capturing the neurophysiological therapeutic effects in terms of changes in the properties of the surface EMG from paretic muscles will require more advanced analysis with tools like recurrence quantification analysis (RQA), which is the focus of the current study.

RQA is a technique for detecting changes in the states of a dynamical systems that does not need any a priori constraint on data size, stationarity, and statistical distribution [32]. RQA parameters are very sensitive in detecting changes in surface EMG due to fatigue [33]. Webber et al. [34] showed that small changes in surface EMG can be detected by RQA where the parameter-\%Det reflects the deterministic attributes of the signal whereas the parameter-\%Rec reflects the recurrence of the signal. RQA parameters have been validated as means for fatigue assessment with potential advantages such as the higher sensitivity to neurophysiological changes in the muscle status [35]. Therefore, the objective of this study was to investigate the effects of NMES-assisted gait training with two different command sources-switch-trigger or EMGtrigger-on two RQA parameters-the percentage of recurrence (\%Rec) and determinism (\%Det), which were extracted from the surface EMG during fatiguing contractions of the paretic muscle in 12 chronic stroke survivors.

\section{Methods \\ Subjects}

Ten male and two female hemiplegic chronic ( $>6$ months post-stroke) stroke survivors (age: 56-75 years) volunteered for this study. Informed consent was obtained from all the subjects before their participation. The study was approved by the Institutional Review Board of Max Superspeciality Hospitals Saket, New Delhi, India. The clinical inclusion and exclusion criteria are tabulated in Table 1 , which were evaluated by a consulting neurologist.

\section{Experimental setup}

Experimental setup and the signal flow are shown in the top panel of Figure 1. Surface EMG signals were 


\begin{tabular}{|c|c|}
\hline Inclusion criteria & Exclusion criteria \\
\hline Age $21-80$ years & Brainstem stroke \\
\hline $\begin{array}{l}>6 \text { months from a first clinical non- } \\
\text { hemorrhagic or hemorrhagic stroke }\end{array}$ & Epilepsy \\
\hline Medically stable & $\begin{array}{l}\text { Severely impaired cognition and } \\
\text { communication }\end{array}$ \\
\hline $\begin{array}{l}\text { Unilateral lower extremity hemi- } \\
\text { paresis }\end{array}$ & History of peroneal nerve injury \\
\hline $\begin{array}{l}\text { Able to ambulate } 16 \text { feet }(5 \mathrm{~m}) \\
\text { continuously with minimal } \\
\text { assistance or less, without the use } \\
\text { of an ankle foot orthosis (AFO) }\end{array}$ & $\begin{array}{l}\text { History of Parkinson's, spinal cord } \\
\text { injury, traumatic brain injury, } \\
\text { multiple sclerosis, uncontrolled } \\
\text { seizure disorder }\end{array}$ \\
\hline $\begin{array}{l}\text { AFO is clinically indicated (foot- } \\
\text { drop during ambulation) }\end{array}$ & $\begin{array}{l}\text { Uncompensated hemi-neglect } \\
\text { (extinguishing to double } \\
\text { simultaneous stimulation) }\end{array}$ \\
\hline $\begin{array}{l}\text { Electrical stimulation of the paretic } \\
\text { ankle dorsiflexors produces ankle } \\
\text { dorsiflexion to neutral without pain }\end{array}$ & $\begin{array}{l}\text { Edema of the paretic lower } \\
\text { extremity }\end{array}$ \\
\hline $\begin{array}{l}\text { Full voluntary dorsiflexion of the } \\
\text { contralateral ankle }\end{array}$ & $\begin{array}{l}\text { Absent sensation of lower leg and } \\
\text { foot }\end{array}$ \\
\hline \multirow[t]{4}{*}{$\begin{array}{l}\text { Skin intact on hemiparetic lower } \\
\text { extremity }\end{array}$} & $\begin{array}{l}\text { History of cardiac arrhythmias with } \\
\text { hemodynamic instability }\end{array}$ \\
\hline & $\begin{array}{l}\text { Cardiac pacemaker or other } \\
\text { implanted electronic system }\end{array}$ \\
\hline & $\begin{array}{l}\text { Botulinum toxin injections to any } \\
\text { lower extremity muscle in the last } \\
3 \text { months }\end{array}$ \\
\hline & $\begin{array}{l}\text { Evidence of deep venous } \\
\text { thrombosis or thromboembolism }\end{array}$ \\
\hline
\end{tabular}

collected from medial gastrocnemius (MG) muscle using $\mathrm{Ag} / \mathrm{AgCl}$ electrodes with 2-cm inter-electrode distance following the SENIAM guidelines [36]. The surface EMG signals were amplified and low-pass filtered (antialiasing, frequency cutoff $=1000 \mathrm{~Hz}$ ) with custom-made amplifier before being sampled at $2400 \mathrm{~Hz}$ by 16-bit data acquisition system (NI USB-6215, National Instruments, USA). The data acquisition system also sampled the heel-switch signal to monitor heel-rise and heel-strike of the paretic foot. The digital output of the data acquisition system activated a switching circuit (clamp) by a trigger pulse that disconnected the EMG electrode inputs from the amplifier and connected them to the common ground electrode from heel-rise to heel-strike when the stimulation was delivered. The gain of the custom-made amplifier was set to prevent saturation at the maximum muscle activity. The control signal for triggering NMES - the linear envelope (LE) - was computed after digitally band-pass filtering (5th order Butterworth, $3 \mathrm{~dB}$ bandwidth $=10-500 \mathrm{~Hz}$ ), then full-wave rectification and then low-pass filtering (5th order Butterworth, $3 \mathrm{~dB}$ frequency cutoff $=3 \mathrm{~Hz}$ ) the sampled EMG signal. Visual biofeedback of the control signal was provided to the subject on a computer monitor (bottom panel of Figure 1) while the subjects learned to trigger the stimulation with surface EMG from their paretic MG muscle.

After achieving proficiency in EMG-triggered FES, the subjects in the EMG-triggered group (see the next section) walked with single-channel transcutaneous NMES device (ODFS Pace, Odstock, UK) which delivered electrical stimulation using saline-soaked sponge electrodes to activate tibialis anterior of the paretic leg during swing-phase of the gait cycle. The stimulation and recording electrodes were attached to a customized sleeve as shown in Figure 2, which was worn by the subject during each visit for gait training.

\section{NMES intervention}

The subjects were randomly allocated to one of the two groups: GROUP EMG-triggered or GROUP Switch-triggered, as explained below.

\section{Group EMG-triggered}

In this group, the subjects received EMG-triggered transcutaneous NMES-assisted gait training on treadmill for $1 \mathrm{~h}$, five times a week for 2 weeks. The NMES was triggered at the heel-rise of the paretic side that was detected with a heel-switch only if the LE of the MG crossed $75 \%$ of the MVC during preceding doublesupport (push-off) phase of the gait. Before walking with EMG-triggered transcutaneous NMES, the subjects were trained to activate paretic MG muscle for push-off using biofeedback of the LE control signal in order to trigger the NMES device.

\section{Group switch-triggered}

In this group, the subjects received heel-switch-triggered transcutaneous NMES-assisted gait training on treadmill for $1 \mathrm{~h}$, five times a week for 2 weeks. The NMES was triggered at the heel-rise of the paretic side that was detected with a heel-switch.

In both the groups, NMES produced muscle contractions that mimic normal voluntary ankle movement, lifting the foot off the ground and improving gait, by applying electrical pulses to the common peroneal nerve through the skin surface. Stimulation electrodes were placed over the nerve using a textile sleeve (Figure 2), which were connected by leads to the NMES device (ODFS Pace, Odstock, UK).

\section{Baseline and post-intervention RQA}

During the baseline and post-intervention surface EMG assessment, subjects were asked to stand steady on their toes for a total of $10 \mathrm{~s}$ with heel-rise of both their feet while the surface EMG was recorded from fatiguing bilateral MG muscles. The EMG signals were amplified and low-pass filtered (anti-aliasing, frequency cutoff $=1000 \mathrm{~Hz}$ ) 

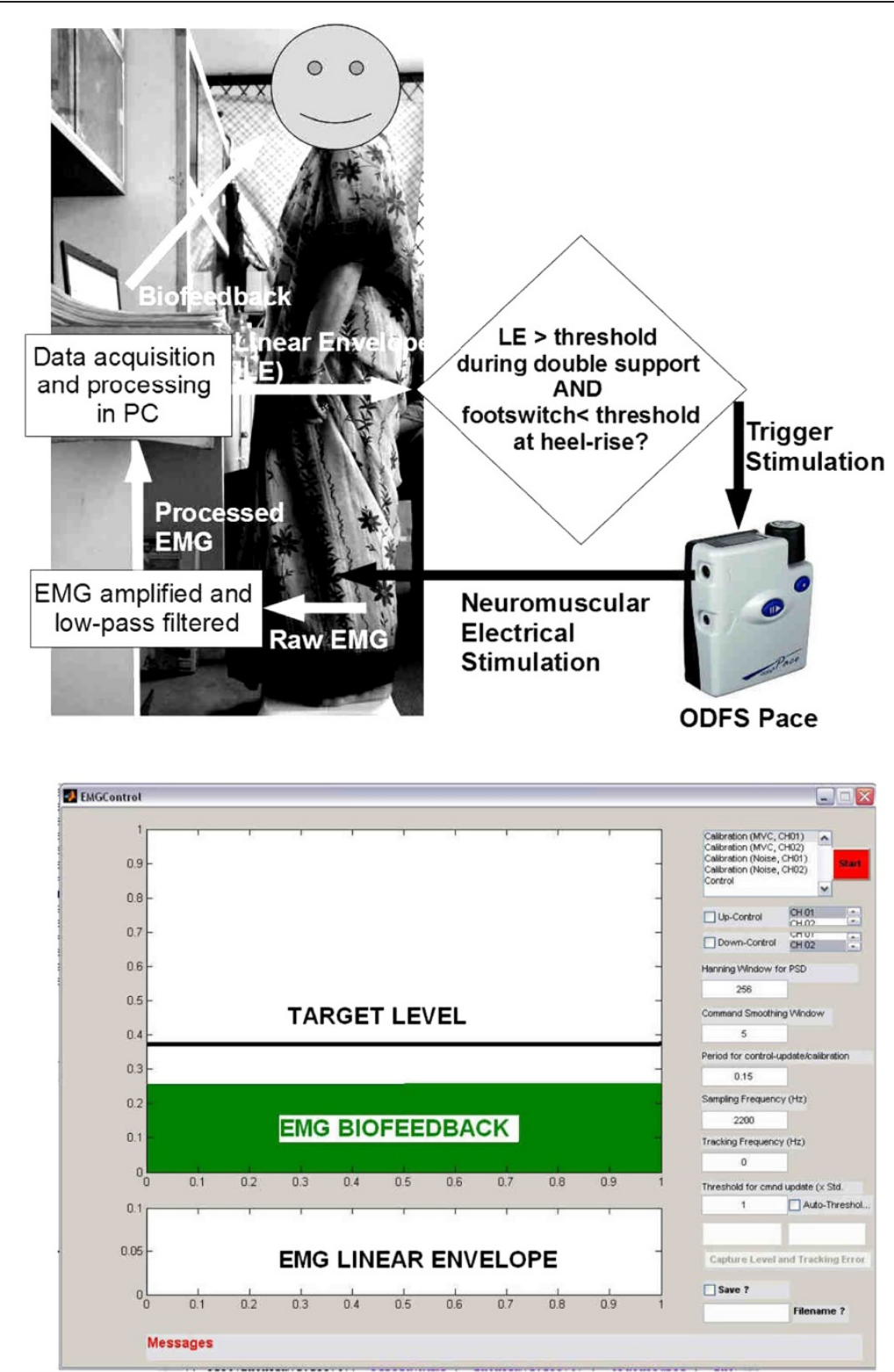

Figure 1 Top panel: Experimental setup for EMG-triggered NMES training. The signal flow is overlaid where the raw surface EMG was collected from MG muscle, which was amplified and low-pass filtered (anti-aliasing, frequency cutoff $=1000 \mathrm{~Hz}$ ) before being sampled at $2400 \mathrm{~Hz}$ by 16-bit data acquisition system (NI USB-6215, National Instruments, USA) in a personal computer (PC). The LE was computed after digitally band-pass filtering (5th order Butterworth, $3 \mathrm{~dB}$ bandwidth $=10-500 \mathrm{~Hz}$ ), then full-wave rectification and the low-pass filtering (5th order Butterworth, $3 \mathrm{~dB}$ frequency cutoff $=3 \mathrm{~Hz}$ ) the sampled EMG signal. Visual biofeedback of the control signal (150 ms sliding window) was provided to the subject on a computer monitor (bottom panel) while the subjects learned to trigger the stimulation by activating their paretic MG muscle by exceeding a subject-specific threshold of 75\% of their maximal voluntary contraction (MVC) in conjunction with heel-rise detected by the heel-switch of the ODFS Pace (Odstock, UK) electrical stimulator. Bottom panel: EMG visual biofeedback for EMG-triggered NMES training.

with custom-made amplifier before being sampled at $2400 \mathrm{~Hz}$ by 16-bit data acquisition system (NI USB-6215, National Instruments, USA). Then the sampled EMG signals were digitally band-pass filtered (5th order Butterworth, $3 \mathrm{~dB}$ bandwidth $=10-500 \mathrm{~Hz}$ ) and re-sampled at $1024 \mathrm{~Hz}$ to be stored for offline analysis. The data acquisition system also sampled bilateral heel-switch signals to monitor heel-rise and heel-strike of both the feet.
The RQA was conducted offline from $9 \mathrm{~s}$ of the surface EMG signal (half seconds of leading and trailing data were discarded) from paretic and non-paretic MG to estimate the parameters-\%Det and \%Rec from adjacent non-overlapping epochs of $1 \mathrm{~s}$ (1,024 data points) for each subject. The first step in RQA was embedding procedure where vectors representing the states of the system were extracted. The phase space was assumed to 


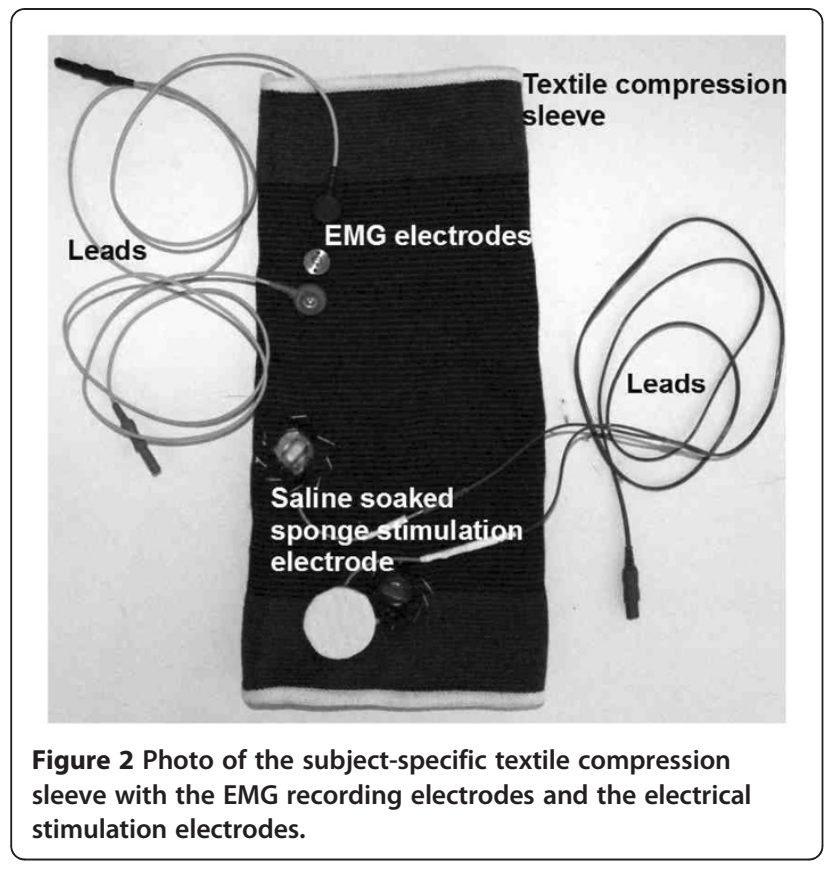

be 15-dimensional [37]. Therefore, $D=15$-dimensional vectors were extracted from $1 \mathrm{~s}$ epoch of the surface EMG signal (i.e., data $=\{s(1), s(2), \ldots, s(1024)\})$ by lagging the signal by an integer number of samples $(d=3$ [35]) as shown below,

$$
\begin{aligned}
& v(1)=\{s(1), s(1+d), s(1+2 \times d), \ldots, s((D-1) \times d)\} \\
& v(2)=\{s(2), s(2+d), s(2+2 \times d), \ldots, s(1+(D-1) \times d)\} \\
& v(N)=\{s(N), s(N+d), s(N+2 \times d), \ldots, s(N+(D-1) \times d)\} \\
& \text { where } N=1024-(D-1) \times d
\end{aligned}
$$

The Euclidean distance matrix $\left(D M_{N x N}\right)$ for the 15dimensional vector was created where the elements were given by

$$
D M_{i, j}=\sum[v(i)-v(j)]^{1 / 2}
$$

The Euclidean distance matrix $\left(D M_{N x N}\right)$ was then normalized $\left(D M_{N x N}^{\text {norm }}\right)$ by the average distance between the vectors

$$
D M_{\mathrm{av}}=\frac{\sum_{i=1}^{N} \sum_{i \neq j}^{N} D M_{i, j}}{N(N-1) / 2}
$$

The recurrence map (RM) was then constructed from $D M_{N x N}^{\text {norm }}$ such that the pixel $p(i, j)$ was $\mathrm{ON}$ if $D M_{N x N}^{\text {norm }} \leq R$ and OFF if $D M_{N x N}^{\text {norm }}>R$, where the threshold was $R=60$ [35]. In the RM, the pixels that were ON were defined as recurrent ( $R e)$ if they share local neighborhoods in higher dimensional space. Therefore, the percentage of the plot occupied by Re points was defined as
\%Rec and the percentage of Re forming lines parallel to diagonal was defined as \%Det. Lines were constituted by two or more points (up to 20) that were diagonally adjacent. Therefore,

$$
\% \text { Det }=\frac{L}{R e} \times 100
$$

where $\mathrm{L}$ was the number of points forming lines.

For each subject, the first-order regression line was computed from nine data points for \%Rec and \%Det during fatiguing contractions of the bilateral MG. The rate of change in \%Rec and \%Det was defined as the slope of the regression line and the initial value as the intercept of the regression line.

\section{Results}

The regression lines capturing the variations in \%Rec and \%Det of the surface EMG during $9 \mathrm{~s}$ of fatiguing contractions of the paretic (affected limb) and nonparetic (unaffected limb) MG muscle before (baseline) FES-assisted gait training are shown in Figure 3 for all the subjects $(N=12)$. The rate of change (slope in $\% /$ second) of \%Rec and \%Det for the paretic MG with their 95\% confidence interval $(\mathrm{CI})$ were $-0.411(\mathrm{CI}=$ -0.614 to -0.208$)$ and $-1.449(\mathrm{CI}=-1.978$ to -0.92$)$, respectively. In comparison, the rate of change (\%/second) of \%Rec and \%Det of surface EMG for the non-paretic MG were $0.193(\mathrm{CI}=0.072-0.313)$ and $1.149(\mathrm{CI}=0.724-$ 1.573), respectively. Therefore, \%Rec and \%Det decreased over $9 \mathrm{~s}$ of fatiguing contractions for the paretic MG while it increased for the non-paretic MG, for all the subjects pooled together $(\mathrm{N}=12)$. The initial values (intercept in \%) of \% Rec and \%Det were $5.978(\mathrm{CI}=4.836-7.12)$ and 60.829 $(\mathrm{CI}=57.852-63.806)$, respectively, for the paretic MG while $1.064(\mathrm{CI}=0.387-1.741)$ and $41.442(\mathrm{CI}=39.052-$ 43.829), respectively, for the non-paretic MG. Therefore, there was a significant difference in the initial values of $\%$ Rec and \%Det for all the subjects pooled together $(N=12)$, which were lower for the non-paretic MG than the paretic MG.

The subjects were randomly divided into two groups of six subjects each-EMG-triggered $(N=6)$ and switch-triggered $(N=6)$. The changes in the slope and intercept of the regression line capturing the variations in \%Rec and \%Det of the surface EMG during $9 \mathrm{~s}$ of fatiguing contractions of the paretic (affected limb) and non-paretic (unaffected limb) before (baseline) and after (post-intervention) FESassisted gait training are tabulated in Additional file 1: Table S2 for each group. The data showed significant changes in the slope and intercept of the regression line for both the RQA parameters-\%Rec and \%Det of the paretic MG for the EMG-trigger group while the switchtrigger group showed no significant changes post- 


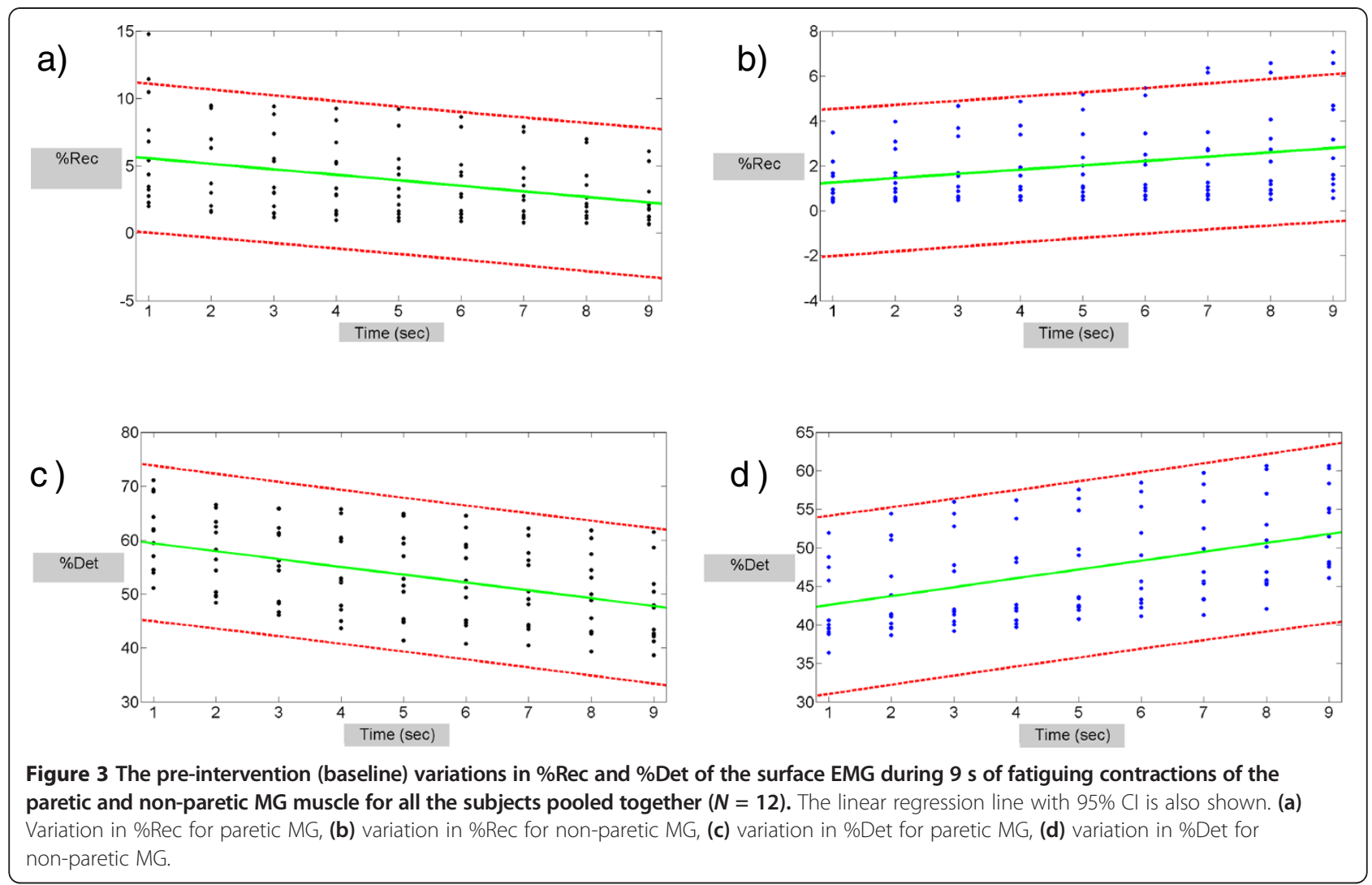

intervention. Specifically, the rate of change of \%Rec during fatiguing contractions increased from $-0.059(\mathrm{CI}=$ -0.088 to -0.031$)$ to $-0.008(\mathrm{CI}=-0.017$ to -0.0006$)$ while that for \%Det increased from $-1.625(\mathrm{CI}=-2.371$ to -0.88$)$ to $-0.4426(\mathrm{CI}=-0.913$ to -0.028$)$ for the EMG-trigger group. Also, the initial value of \%Rec decreased from 7.727 $(\mathrm{CI}=6.139-9.315)$ to $2.302(\mathrm{CI}=1.807$ to -2.797$)$ while that for \%Det decreased from 64.164 (CI = $59.968-68.359)$ to $49.901(\mathrm{CI}=47.252-52.55)$ for the EMG-trigger group. The corresponding values for the switch-trigger group remained statistically unchanged post-intervention.

\section{Discussion}

RQA has been shown to be promising for detecting neurophysiological changes in the muscle due to fatigue or other factors [35]. Farina et al. [35] showed that both the parameters-\%Rec and \%Det-were influenced by the conduction velocity and synchronization of the motor units of the muscle and therefore sensitive to the muscle properties. They also showed that \%Det was highly correlated with the spectral variables and to some extent \%Rec as well, and moreover both the \%Det and \% Rec were more sensitive to muscle fatigue than the spectral variables. The mean spectral frequency (MNF) and \%Det were highly correlated with a negative correlation coefficient close to -1 .
In this study, the parameters from RQA captured the differences in the muscle properties between paretic and non-paretic MG muscle (Figure 3) as well as the changes affected by NMES-assisted gait training interventionsEMG-triggered versus switch-triggered (Additional file 1: Table S2). From the baseline data, it was found that the parameters-\%Rec and \%Det-had a higher initial value for the paretic MG than the non-paretic MG. A higher value for \%Det indicated a lower MNF for the paretic MG when compared to the non-paretic MG. Moreover, the rate of change of \%Rec and \%Det during fatiguing contraction was negative for the paretic MG and positive for the non-paretic MG. A positive rate of change in \% Det indicated a decrease in MNF during fatiguing contractions which is normal [38]; however, a negative rate of change indicated an increase in MNF which may be pathological for the paretic MG. Moreover, the rate of change in \%Rec and \%Det during the fatiguing contractions of the paretic MG increased after NMESassisted gait training for the EMG-trigger group. Felici et al. [39] have shown a higher rate of change in \%Det in weight lifters when compared to the control group during sustained isometric contractions. A significant increase in the rate of change in \%Det during fatiguing contractions may indicate exercise-effect on 
the paretic MG muscle brought by EMG-triggered NMES-assisted gait training. Therefore, we found that our short-duration EMG-triggered FES-assisted gait therapy improved neurophysiological properties of the muscle and a more intensive treatment with duration beyond 2 weeks might have shown additional benefits [40-43].

However, lack of accessible rehabilitation facilities and high cost of rehabilitation at the well-equipped clinics are current challenges in India which usually lead to high-dropout of individuals from rehabilitation therapy following stroke. Such de-conditioned chronic stroke survivor will need to recondition themselves with a gradual increase in the intensity (number of hours per day) and frequency (number of days per week) over the duration of the FES-assisted gait therapy. In fact, about $20 \%$ of stroke survivors suffer muscle atrophy which underlie worsening metabolic fitness in the chronic phase of stroke including gross muscular atrophy, altered muscle molecular phenotype, increased intramuscular area fat, elevated tissue inflammatory markers, and diminished peripheral blood flow dynamics [44]. EMG-triggered FES-assisted gait training may alleviate these debilitating conditions where increased intensity and frequency of rehabilitation may help [40]. In fact recent studies in India on therapeutic benefits of FESassisted gait training in conjunction with conventional physiotherapy have shown the additive effects of FES on reducing spasticity, improving dorsiflexor strength, improving walking ability, and improving metabolic fitness $[41,42]$. However, stroke presents with heterogeneous patient-specific impairments in motor, sensory, tone, visual, perceptual, cognition, aphasia, apraxia, coordination, and equilibrium. Therefore, the functional limitations following stroke are varied, including gait dysfunction, fall risk, limited activities of daily living, difficulties in swallowing, reduced upper extremity function, altered communication, besides others. Based on the residual function of a stroke survivor, the number of muscles that can serve as EMG command source [31], the number of muscles that need FES assistance, intensity, frequency, and duration of the EMG-triggered FES-assisted gait training need to be decided. The ability of a stroke survivor to undergo FES-assisted gait therapy also depends on their cardiovascular and neuromuscular capacity besides psychological factors such as motivation. Also, the FES-assisted gait training should be started as soon as the patient becomes clinically stable since early intervention has shown better functional outcomes, survival rates, and reduced length of required therapy [43]. Therefore, we are currently investigating a home-based rehabilitation model where stroke survivors can use a low-cost EMG-triggered FES device daily. However, the efficacy of such home-based models in affecting therapeutic benefits needs to be validated with neurophysiological testing with advanced computational tools like RQA where parameters like \%Rec and \%Det can non-invasively monitor changes in muscle properties due to NMES-assisted gait training during rehabilitation.

\section{Conclusion}

The overarching goal of NMES-assisted gait training is to facilitate long-term therapeutic changes in muscle properties. However, a sensitive biomarker is needed to capture these neurophysiological changes during motor rehabilitation. In this study, the parameters derived from RQA captured the significant changes in muscle properties brought by EMG-triggered NMES-assisted gait training when compared to switch-triggered NMESassisted gait training. The subjects were forced to volitionally activate the paretic MG muscle to generate the EMG-command signal during EMG-triggered NMESassisted gait training which exercised the paretic MG muscle during a functional task. Therefore, EMGtriggered NMES-assisted gait training could be more effective in providing long-term therapeutic benefit in muscle function than switch-triggered NMES-assisted gait training. This study also provided an understanding of RQA parameters-\%Rec and \%Det, computed from surface EMG of the paretic and non-paretic muscles during fatiguing contractions.

\section{Additional file}

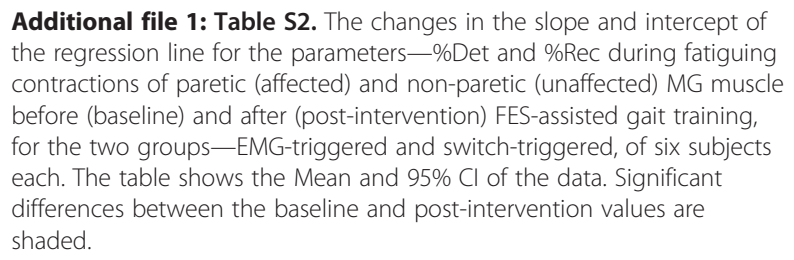

\section{Competing interests}

The authors declare no existence of competing interests during the conduction of this study where the professional judgment concerning patients' welfare and the validity of the research may be influenced by financial gain or personal opinion.

\section{Acknowledgments}

Dr. Dutta (Ph.D.) was supported by the Alexander von Humboldt Foundation, Germany. Drs. Banerjee (P.T.) and Khattar (P.T.) were supported by the Max Superspecialty Hospitals Saket, New Delhi, India. The voluntary help received from the subjects is gratefully acknowledged. The clinical study is registered with the Clinical Trials Registry of India (http://www.ctri.nic.in/Clinicaltrials/ pmaindet2. php?trialid=3821).

\section{Author details}

'Department of Clinical Neurophysiology, Georg-August-University, Goettingen, Germany. ${ }^{2}$ Department of Physiotherapy and Rehabilitation, Max Super Specialty Hospital Saket, New Delhi, India. 


\section{References}

1. World Health Organisation, Preventing Chronic Diseases. A Vital Investment (World Health Organisation, Geneva, Switzerland, 2005)

2. P Dalal, M Bhattacharjee, J Vairale, P Bhat, UN millennium development goals: can we halt the stroke epidemic in India? Ann. Indian Acad. Neurol 10, 130-136 (2007)

3. P Dalal, S Malik, M Bhattacharjee, ND Trivedi, J Vairale, P Bhat, Populationbased stroke survey in Mumbai, India: incidence and 28-day case fatality. Neuroepidemiology 31, 254-261 (2008)

4. SE Sridharan, JP Unnikrishnan, S Sukumaran, PN Sylaja, SD Nayak, PS Sarma, K Radhakrishnan, Incidence, types, risk factors and outcome of stroke in a developing country: the Trivandrum Stroke Registry. Stroke 40, 1212-18 (2009)

5. M Tripathi, D Vibha, Stroke in young in India. Stroke Res. Treatment 2011, 1-6 (2011)

6. B Shah, Development of Sentinel Health Monitoring Centers for Surveillance of Risk Factors for Non-Communicable Disease in India (Report from the Division of Non-communicable Diseases (Indian Council of Medical Research, New Delhi, 2005)

7. B Shah, P Mathur, Workshop Report on Stroke Surveillance in India (Division of noncommunicable Diseases, Indian Council of Medical Research, New Delhi, 2006)

8. MH Granat, DJ Maxwell, ACB Ferguson, KR Lees, JC Barbanel, Peroneal stimulator: evaluation for the correction of spastic foot-drop in hemiplegia. Arch. Phys. Med. Rehabil. 77(1), 19-24 (1996)

9. V Weerdesteyn, M de Niet, HJ van Duijnhoven, AC Geurts, Falls in individuals with stroke. J Rehabil. Res. Dev. 45(8), 1195-1213 (2008)

10. SR Goldberg, FC Anderson, MG Pandy, SL Delp, Muscles that influence knee flexion velocity in double support: implications for stiff-knee gait. J Biomech. 37(8), 1189-1196 (2004)

11. NA Solomon, HA Glick, CJ Russo, J Lee, KA Schulman, Patient preferences for stroke outcomes. Stroke 25, 1721-1725 (1994)

12. H Barbeau, J Fung, The role of rehabilitation in the recovery of walking in the neurological population. Curr. Opin. Neurol. 14(6), 735-740 (2001)

13. R Merletti, A Andina, M Galante, I Furlan, Clinical experience of electronic peroneal stimulators in 50 hemiparetic patients. Scand. J Rehabil. Med. 11 (3), 111-121 (1979)

14. U Bogataj, N Gros, M Malezic, B Kelih, M Kilajic, R Acimovic, Restoration of gait during two to three weeks of therapy with multichannel electrical stimulation. Phys. Ther. 69(5), 319-327 (1989)

15. PN Taylor, JH Burridge, AL Dunkerley, DE Wood, JA Norton, C Singleton, ID Swain, Clinical use of the Odstock dropped foot stimulator: Its effect on speed and effort of walking. Arch. Phys. Med. Rehabil. 80(12), 1577-1583 (1999)

16. LR Sheffler, MT Hennessey, GG Naples, J Chae, Peroneal nerve stimulation versus an ankle foot orthosis for correction of footdrop in stroke: impact on functional ambulation. Neurorehabil. Neural Repair 20(3), 355-360 (2006)

17. Al Kottink, HJ Hermens, AV Nene, MJ Tenniglo, CG Groothuis-Oudshoorn, MJ IJzerman, Therapeutic effect of an implantable peroneal nerve stimulator in subjects with chronic stroke and footdrop: a randomized controlled trial. Phys. Ther 88(4), 437-448 (2008)

18. JH Burridge, PN Taylor, ID Swain, The effects of common peroneal stimulation on the effort and speed of walking: a randomized controlled trial with chronic hemiplegic patients. Clin. Rehabil. 11(3), 201-210 (1997)

19. JH Burridge, M Ladouceur, Clinical and therapeutic applications of neuromuscular stimulation: a review of current use and speculation into future developments. Neuromodulation 4, 147-155 (2001)

20. JM Hausdorff, $\mathrm{H}$ Ring, Effects of a new radio frequency controlled neuroprosthesis on gait symmetry and rhythmicity in patients with chronic hemiparesis. Am. J Phys. Med. Rehabil. 87(1), 4-13 (2007)

21. JJ Daly, J Zimbelman, KL Roenigk, JP McCabe, JM Rogers, K Butler, R Burdsall, JP Holcomb, EB Marsolais, RL Ruff, Recovery of coordinated gait: randomized controlled stroke trial of functional electrical stimulation (FES) versus no FES, with weight-supported treadmill and over-ground training. Neurorehabil. Neural Repair 25(7), 588-96 (2011)

22. JJ Daly, RL Ruff, Feasibility of combining multi-channel functional neuromuscular stimulation with weight-supported treadmill training. J Neurol. Sci. 225, 105-115 (2004)

23. R. Kobetic, RJ Triolo, EB Marsolais, Muscle selection and walking performance of multichannel FES systems for ambulation in paraplegia. IEEE Trans. Rehabil. Eng. 5(1), 23-29 (1997)
24. A Dutta, R Kobetic, RJ Triolo, Ambulation after incomplete spinal cord injury with EMG-triggered functional electrical stimulation. IEEE Trans. Biomed. Eng. 55(2 Pt 1), 791-794 (2008)

25. A Dutta, R Kobetic, RJ Triolo, Gait initiation with electromyographically triggered electrical stimulation in people with partial paralysis. J Biomech. Eng. 131(8), 0810021-9 (2009)

26. L Vodovnik, C Long, JB Reswick, A Lippay, D Starbuck, Myo-electric control of paralyzed muscles. IEEE Trans. Biomed. Eng. 12(3), 169-172 (1965)

27. DA Bolton, JH Cauraugh, HA Hausenblas, Electromyogram-triggered neuromuscular stimulation and stroke motor recovery of arm/hand functions: a meta-analysis. J Neurol. Sci. 223(2), 121-127 (2004)

28. G Francisco, J Chae, H Chawla, S Kirshblum, R Zorowitz, G Lewis, S Pang, Electromyogram-triggered neuromuscular stimulation for improving the arm function of acute stroke survivors: a randomized pilot study. Arch. Phys. Med. Rehabil. 79(5), 570-575 (1998)

29. J Cauraugh, K Light, S Kim, M Thigpen, M Beh Thigpen, A Behrman, Chronic motor dysfunction after stroke: recovering wrist and finger extension by electromyography triggered neuromuscular stimulation. Stroke 31(6), 13601364 (2000)

30. RW Fields, Electromyographically triggered electric muscle stimulation for chronic hemiplegia. Arch. Phys. Med. Rehabil. 68(7), 407-414 (1987)

31. A Dutta, R Kobetic, RJ Triolo, An objective method for selecting command sources for myoelectrically controlled lower extremity neuroprostheses. J Rehabil. Res. Dev. 48(8), 935-948 (2011)

32. JP Eckmann, SO Kamphorst, D Ruelle, Recurrence plots of dynamical systems. Europhys. Lett 4, 973-977 (1987)

33. S Ikegawa, M Shinohara, T Fukunaga, JP Zbilut, CL Webber Jr., Nonlinear time-course of lumbar muscle fatigue using recurrence quantifications. Biol. Cybern. 82, 373-382 (2000)

34. CL Webber Jr., M.A. Schmidt, JM Walsh, Influence of isometric loading on biceps EMG dynamics as assessed by linear and nonlinear tools. J Appl. Physiol 78, 814-822 (1995)

35. D Farina, L Fattorini, F Felici, G Filligoi, Nonlinear surface EMG analysis to detect changes of motor unit conduction velocity and synchronization. J Appl. Physiol 93, 1753-1763 (2002)

36. R Merletti, $\mathrm{H}$ Hermens, Introduction to the special issue on the SENIAM European Concerted Action. J Electromyogr. Kinesiol. 10(5), 283-6 (2000)

37. G. Filligoi, F. Felici, Detection of hidden rhythms in surface EMG signals with a nonlinear time-series tool. Med. Eng. Phys 21, 439-448 (1999)

38. R Merletti, M Knaflitz, CJ De Luca, Myoelectric manifestations of fatigue in voluntary and electrically elicited contractions. J Appl. Physiol 69, 1810-1820 (1990)

39. F Felici, A Rosponi, P Sbriccoli, GC Filligoi, L Fattorini, M Marchetti, Linear and nonlinear analysis of surface electromyograms in weightlifters. Eur. J Appl. Physiol 84, 337-342 (2001)

40. G Kwakkel, RC Wagenaar, TW Koelman, GJ Lankhorst, JC Koetsier, Effects of intensity of rehabilitation after stroke. A research synthesis. Stroke 28(8), 1550-1556 (1997)

41. SK Sabut, PK Lenka, R Kumar, M Mahadevappa, Effect of functional electrical stimulation on the effort and walking speed, surface electromyography activity, and metabolic responses in stroke subjects. J Electromyogr. Kinesiol. 20(6), 1170-1177 (2010)

42. SK Sabut, C Sikdar, R Kumar, M Mahadevappa, Functional electrical stimulation of dorsiflexor muscle: effects on dorsiflexor strength, plantarflexor spasticity, and motor recovery in stroke patients. NeuroRehabilitation 29(4), 393-400 (2011)

43. M Musicco, L Emberti, G Nappi, C Caltagirone, Early and long-term outcome of rehabilitation in stroke patients: the role of patient characteristics, time of initiation, and duration of interventions. Arch. Phys. Med. Rehabil. 84(4), 551-558 (2003)

44. FM Ivey, RF Macko, AS Ryan, CE Hafer-Macko, Cardiovascular health and fitness after stroke. Topics Stroke Rehabil. Winter 12(1), 1-16 (2005)

doi:10.1186/1687-6180-2012-153

Cite this article as: Dutta et al:: Nonlinear analysis of electromyogram following gait training with myoelectrically triggered neuromuscular electrical stimulation in stroke survivors. EURASIP Journal on Advances in Signal Processing 2012 2012:153. 